Document downloaded from:

http://hdl.handle.net/10251/55081

This paper must be cited as:

Bonet Solves, JA.; Fernandez Rosell, C. (2014). The range of the restriction map for a multiplicity variety in Hörmander algebras of entire functions. Mediterranean Journal of Mathematics. 11(2):643-652. doi:10.1007/s00009-013-0318-5.

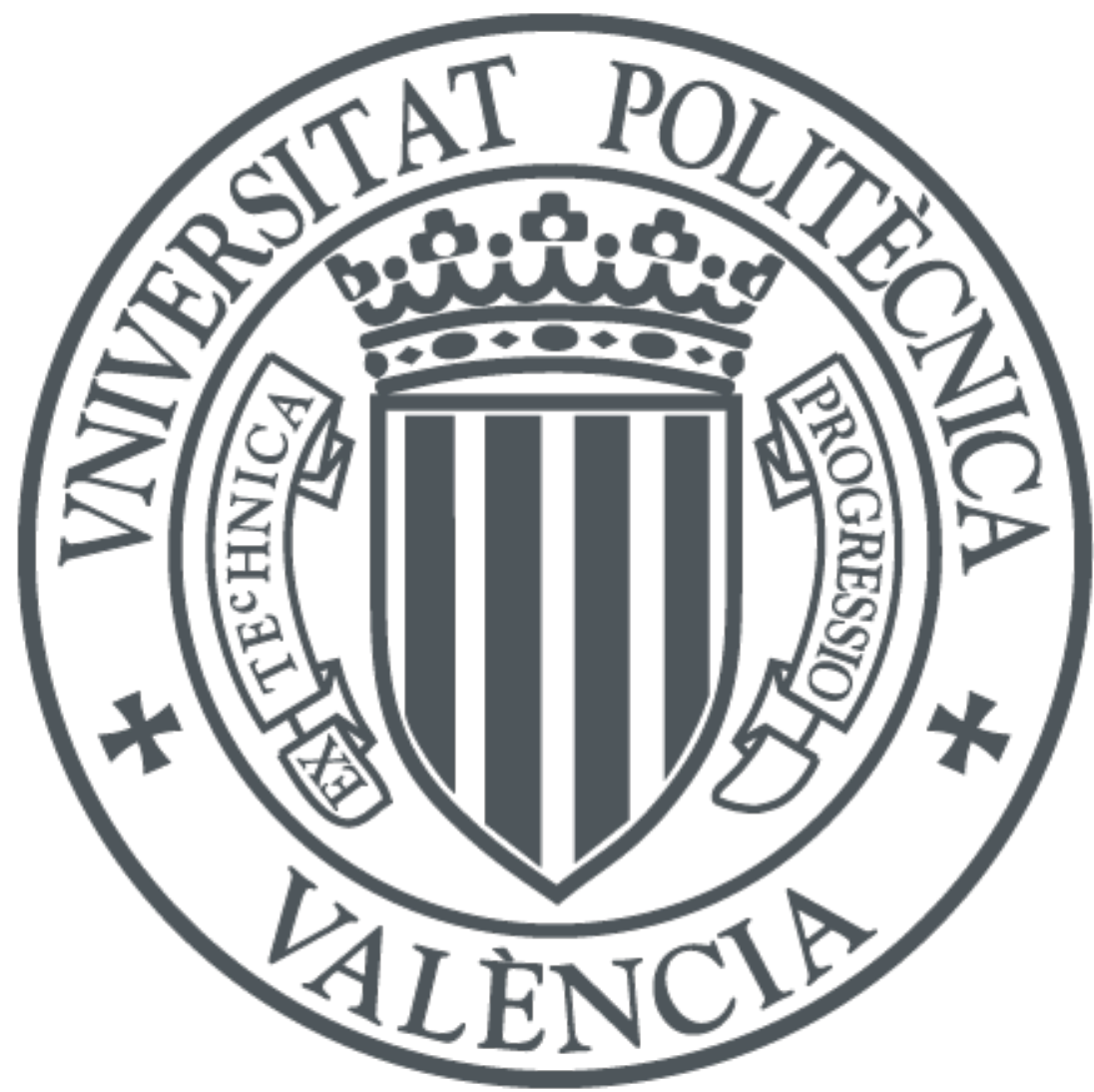

The final publication is available at

The final publication is available at Springer via http://dx.doi.org/10.1007/s00009-013-0318-5

Copyright Springer Verlag (Germany)

Additional Information 


\title{
The range of the restriction map for a multiplicity variety in Hörmander algebras of entire functions
}

\author{
José Bonet, and Carmen Fernández
}

\begin{abstract}
Characterizations of interpolating multiplicity varieties for Hörmander algebras $A_{p}(\mathbb{C})$ and $A_{p}^{0}(\mathbb{C})$ of entire functions were obtained by Berenstein, Li and Vidras [3] and [4] for a radial subharmonic weight $p$ with the doubling property. In this note we consider the case when the multiplicity variety is not interpolating, we compare the range of the associated restriction map for two weights $q \leq p$ and investigate when the range of the restriction map on $A_{p}(\mathbb{C})$ or $A_{p}^{0}(\mathbb{C})$ contains certain subspaces associated in a natural way with the smaller weight $q$.
\end{abstract}

\section{Introduction and preliminaries}

In this paper we consider the following question on interpolation for radial Hörmander algebras of entire functions on the complex plane: Assume that a multiplicity variety $V$ is not necessarily interpolating on a radial Hörmander algebra $A_{p}(\mathbb{C})\left(\operatorname{resp} . A_{p}^{0}(\mathbb{C})\right)$, in this case the restriction map (see the precise definitions below) needs not be surjective. Let $q \leq p$ be another weight. We investigate conditions to ensure that the sequence space canonically associated with the interpolation for $A_{q}(\mathbb{C})\left(\right.$ resp. $A_{q}^{0}(\mathbb{C})$ ) is contained in the range of the restriction map defined on the bigger space $A_{p}(\mathbb{C})$ (resp. $A_{p}^{0}(\mathbb{C})$ ). Our results complement recent work by Ounaïes [18] and Massaneda, Ortega-Cerdà and Ounaïes [13].

A function $p: \mathbb{C} \rightarrow] 0, \infty[$ is called a weight function if it satisfies the following properties: (w1) $p$ is continuous and subharmonic, (w2) $p$ is radial, that is, $p(z)=p(|z|), z \in \mathbb{C}$, (w3) $\log \left(1+|z|^{2}\right)=o(p(z))$ as $|z| \rightarrow \infty$; and (w4) $p$ is doubling, i.e. $p(2 z)=O(p(z))$ as $|z| \rightarrow \infty$. We use here and in the rest of the paper Landau's notation of little $o$-growth and capital $O$-growth. The space of entire functions is denoted by $\mathcal{H}(\mathbb{C})$. It is a Fréchet space when it is endowed with the topology of uniform convergence on the compact sets in $\mathbb{C}$.

Given a weight $p$, we define the following weighted spaces of entire functions (see e.g. [5], [2], [3], [4]):

$$
A_{p}(\mathbb{C}):=\left\{f \in \mathcal{H}(\mathbb{C}): \text { there is } A>0: \sup _{z \in \mathbb{C}}|f(z)| \exp (-A p(z))<\infty\right\},
$$

endowed with the inductive limit topology, for which it is a (DFN)-algebra (cf. [14]); and

2010 Mathematics Subject Classification. Primary: 46E10, secondary: 30D15, 30D20, 30E05, 46A04

Key words and phrases. Discrete interpolating varieties; entire functions; growth conditions; weighted spaces of entire functions

Acknowledgement. This research was partially supported by MEC and FEDER Project MTM2010-15200. 


$$
A_{p}^{0}(\mathbb{C}):=\left\{f \in \mathcal{H}(\mathbb{C}): \text { for all } \varepsilon>0: \sup _{z \in \mathbb{C}}|f(z)| \exp (-\varepsilon p(z))<\infty\right\},
$$

endowed with the projective topology, for which it is a nuclear Fréchet algebra (cf. [15]).

For undefined notation on complex analysis we refer the reader to [1] and [2], and for functional analysis to $[16]$.

Clearly $A_{p}^{0}(\mathbb{C}) \subset A_{p}(\mathbb{C})$. Condition (w3) implies that $A_{p}^{0}(\mathbb{C})$ contains the polynomials, and condition (w4) implies that the spaces are stable under differentiation. Weighted algebras of entire functions of this type have been considered since the work of Berenstein and Taylor [5] by many authors; see e.g. [2] and the references therein. Braun, Meise and Taylor studied in [8], [14] and [15] the structure of (complemented) ideals in these algebras. A characterization of closed principal ideals in non-radial Hörmander algebras in of holomorphic functions of several variables is provided in [7].

Here are some examples: When $p(z)=|z|^{s}$, then $A_{p}(\mathbb{C})$ consists of all entire functions of order $s$ and finite type or order less than $s$; and $A_{p}^{0}(\mathbb{C})$ is the space of all entire functions of order at most $s$ and type 0 . For $s=1, A_{p}(\mathbb{C})$ is the space of all entire functions of exponential type, and $A_{p}^{0}(\mathbb{C})$ is the space of entire functions of infraexponential type.

Throughout this paper $V=\left\{\left(z_{k}, m_{k}\right) \mid k \in \mathbb{N}\right\}$ denotes a multiplicity variety, that is, a sequence of different points $\left(z_{k}\right)_{k}$ with $\lim _{k \rightarrow \infty}\left|z_{k}\right|=\infty$ and a sequence $\left(m_{k}\right)_{k}$ of positive integers corresponding to the multiplicities at the points $z_{k}$.

By Weierstrass interpolation theorem (see e.g. [1]), the restriction map

$$
R_{V}: \mathcal{H}(\mathbb{C}) \rightarrow \prod_{k \in \mathbb{N}} \mathbb{C}^{m_{k}}, \quad R_{V}(g):=\left(\left(\frac{g^{(l)}\left(z_{k}\right)}{l !}\right)_{0 \leq l<m_{k}}\right)_{k}
$$

is surjective.

We associate with a multiplicity variety $V=\left\{\left(z_{k}, m_{k}\right) \mid k \in \mathbb{N}\right\}$ and a weight $p$ the following sequence spaces

$$
A_{p}(V):=\left\{a=\left(a_{k, l}\right) \in \prod_{k \in \mathbb{N}} \mathbb{C}^{m_{k}} \mid \text { there is } B>0: \sup _{k \in \mathbb{N}} \sum_{l=0}^{m_{k}-1}\left|a_{k, l}\right| \exp \left(-B p\left(z_{k}\right)\right)<\infty\right\},
$$

endowed with the inductive limit topology; and

$$
A_{p}^{0}(V):=\left\{a=\left(a_{k, l}\right) \in \prod_{k \in \mathbb{N}} \mathbb{C}^{m_{k}} \mid \text { for all } \varepsilon>0: \sup _{k \in \mathbb{N}} \sum_{l=0}^{m_{k}-1}\left|a_{k, l}\right| \exp \left(-\varepsilon p\left(z_{k}\right)\right)<\infty\right\},
$$

endowed with the projective topology, for which it is a Fréchet space.

It is well-known that $R_{V}\left(A_{p}(\mathbb{C})\right) \subset A_{p}(V)$ and $R_{V}\left(A_{p}^{0}(\mathbb{C})\right) \subset A_{p}^{0}(\mathbb{C})$; see [5], [2], [3], [4]. A multiplicity variety is called interpolating for $A_{p}(\mathbb{C})\left(\right.$ resp. for $\left.A_{p}^{0}(\mathbb{C})\right)$ if $R_{V}\left(A_{p}(\mathbb{C})\right)=A_{p}(V)$ $\left(\right.$ resp. $\left.R_{V}\left(A_{p}^{0}(\mathbb{C})\right)=A_{p}^{0}(\mathbb{C})\right)$. After the seminal work by Berenstein and Taylor [5], a geometric characterization of the interpolating varieties for $A_{p}(\mathbb{C})$ (resp. for $A_{p}^{0}(\mathbb{C})$ ) was obtained by Berenstein and Li [3] (resp. Berenstein, Li and Vidras [4]). An easier proof was presented by Ounaïes [17] (see also [11]). These geometric characterizations were formulated in terms of 
the counting function and the integrated counting function of the multiplicity variety $V$, that are defined as follows: For $z \in \mathbb{C}$ and $r>0$, we set

$$
n_{V}(z, r):=\sum_{\left|z-z_{k}\right| \leq r} m_{k}
$$

and

$$
N_{V}(z, r):=\int_{0}^{r} \frac{n_{V}(z, t)-n_{V}(z, 0)}{t} d t+n_{V}(z, 0) \log r .
$$

Here is the geometric characterizations of interpolating varieties mentioned above. We include them explicitly, since they will be used repeatedly in the paper.

Theorem 1.1 Let $V=\left\{\left(z_{k}, m_{k}\right) \mid k \in \mathbb{N}\right\}$ be a multiplicity variety and let $p$ be a weight.

(a) (Berenstein, Li [3]) $V$ is interpolating for $A_{p}(\mathbb{C})$ if and only if

(i) $N_{V}(r, 0)=O(p(r))$ as $r \rightarrow \infty$, and

(ii) $N_{V}\left(z_{k},\left|z_{k}\right|\right)=O\left(p\left(z_{k}\right)\right)$ as $k \rightarrow \infty$.

(b) (Berenstein, Li and Vidras [4]) $V$ is interpolating for $A_{p}^{0}(\mathbb{C})$ if and only if

(i) $N_{V}(r, 0)=o(p(r))$ as $r \rightarrow \infty$, and

(ii) $N_{V}\left(z_{k},\left|z_{k}\right|\right)=o\left(p\left(z_{k}\right)\right)$ as $k \rightarrow \infty$.

This result has been extended in different directions. Massaneda, Ortega-Cerdà and Ounaïes [12] gave a geometric description of the interpolating varieties for the algebra of Fourier transforms of distributions and Beurling ultradistributions with compact support on the real line, improving earlier results by Ehrenpreis, Malliavin and Squires. This corresponds to spaces of type $A_{p}(\mathbb{C})$ for non radial weights $p$. The case of Roumieu ultradistributions was studied by Zioło [21]. The arguments of Berenstein and Li [3] were simplified by Hartmann and Massaneda [11] and by Ounaïes [17] using Hörmander's $L^{2}$ estimates for the $\bar{\partial}$ equation, treating also weights that are radial but not doubling. In [18] Ounaïes uses divided differences to characterize those sequences that are in the range $R_{V}\left(A_{p}(\mathbb{C})\right)$ of the restriction map $R_{V}$ when the multiplicity variety satisfies the assumption (a) (i) in Theorem 1.1. This work was continued later by her jointly with Massaneda and Ortega-Cerdà in [13]. Traces of functions in Bargmann-Fock spaces on lattices of critical density are investigated by Buckley, Massaneda and Ortega-Cerdà in [10]. We also refer the reader to the references in the aforementioned papers.

Let us formulate precisely the problems considered in this note. Let $q$ and $p$ two weights such that $q(z)=O(p(z))$ as $|z| \rightarrow \infty$. In this case, $A_{q}(\mathbb{C}) \subset A_{p}(\mathbb{C})$ and $A_{q}(V) \subset A_{p}(V)$. By Theorem 1.1 (a), if $V$ is interpolating for $A_{q}(\mathbb{C})$, then it is interpolating for $A_{p}(\mathbb{C})$. Now assume that the two weights are really different, i.e. $q(z)=o(p(z))$ as $|z| \rightarrow \infty$. Is there a multiplicity variety $V$ such that $V$ is interpolating for $A_{p}(\mathbb{C})$, but not for $A_{q}(\mathbb{C})$ ? The answer is affirmative as we show in Theorem 2.4. As a complement to this result, we prove that if the range $R_{V}\left(A_{p}(\mathbb{C})\right)$ contains the sequence space $A_{q}(V)$ associated with the weight $q$, then $V$ is interpolating for $A_{p}(\mathbb{C})$. In other words, if every sequence in the space $A_{q}(V)$ can be interpolated by a function in $A_{p}(\mathbb{C})$, then every sequence in the larger space $A_{p}(V)$ can be interpolated by a function in $A_{p}(\mathbb{C})$. See Proposition 2.2. A somewhat similar phenomenon 
appeared in the range of convolution operators on spaces of non quasianalytic functions; cf. $[6]$. The corresponding results for the Fréchet spaces $A_{q}^{0}(\mathbb{C}) \subset A_{p}^{0}(\mathbb{C})$ are investigated in Section 3.

\section{Subspaces of the restriction map on $A_{p}(\mathbb{C})$}

Lemma 2.1 Let $V=\left\{\left(z_{k}, m_{k}\right) \mid k \in \mathbb{N}\right\}$ a multiplicity variety and let $q$ and $p$ be weights such that $q(z)=O(p(z))$ as $|z| \rightarrow \infty$. If the restriction map $R_{V}$ satisfies $A_{q}(V) \subset R_{V}\left(A_{p}(\mathbb{C})\right)$, then there is a sequence $\left(f_{k}\right)_{k} \subset A_{p}(\mathbb{C})$ such that $f_{k}^{(l)}\left(z_{j}\right)=0,0 \leq l<m_{j}$, for each $k$ and $j$, except $f_{k}^{\left(m_{k}-1\right)}\left(z_{k}\right) /\left(m_{k}-1\right) !=1$; and there is a constant $A>0$ such that $\left|f_{k}(z)\right| \leq A \exp (A p(z))$ for each $z \in \mathbb{C}$ and each $k \in \mathbb{N}$.

Proof. The space $A_{p}(\mathbb{C})$ is the inductive limit of the sequence of Banach spaces $\left(A_{p, n}(\mathbb{C})\right)_{n}$ defined as follows

$$
\left.A_{p, n}(\mathbb{C})\right):=\left\{f \in \mathcal{H}(\mathbb{C})\left|\|f\|_{p, n}:=\sup _{z \in \mathbb{C}}\right| f(z) \mid \exp (-n p(z))<\infty\right\} .
$$

The Banach sequence space

$$
X_{1}:=\left\{a=\left(a_{k, l}\right) \in \prod_{k \in \mathbb{N}} \mathbb{C}^{m_{k}}\left|\quad\|a\|_{1}:=\sup _{k \in \mathbb{N}} \sum_{l=0}^{m_{k}-1}\right| a_{k, l} \mid \exp \left(-q\left(z_{k}\right)\right)<\infty\right\}
$$

is continuously included in the space $A_{q}(V)$. By assumption $X_{1} \subset A_{q}(V) \subset R_{V}\left(A_{p}(\mathbb{C})\right) \subset$ $\left.\cup_{n \in \mathbb{N}} R_{V}\left(A_{p, n}(\mathbb{C})\right)\right)$. Since the inclusion $i: A_{q}(V) \rightarrow A_{p}(V)$ is continuous, we can apply Grothendieck factorization theorem [16, Theorem 24.33] to find $n \in \mathbb{N}$ such that $X_{1} \subset$ $R_{V}\left(A_{p, n}(\mathbb{C})\right)$ and moreover, the unit ball $B_{1}$ of $X_{1}$ is contained in the image by $R_{V}$ of a bounded subset $C$ of $A_{p, n}(\mathbb{C})$. The sequence $\left(a^{k}\right)_{k}$ defined by $a^{k}:=\left(a_{j, l}^{k}\right), a_{j, l}^{k}=0,0 \leq l<m_{j}$, for each $k$ and $j$, except $a_{m_{k}-1, k}^{k}=1$, is contained in $B_{1}$. Since $B_{1} \subset R_{V}(C)$, for each $k \in \mathbb{N}$ we can find $f_{k} \in C$ such that $R_{V}\left(f_{k}\right):=a^{k}$. Since $C$ is bounded in $A_{p, n}(\mathbb{C})$, it is easy to see that the sequence $\left(f_{k}\right)_{k}$ satisfies all the required conditions.

Proposition 2.2 Let $V=\left\{\left(z_{k}, m_{k}\right) \mid k \in \mathbb{N}\right\}$ a multiplicity variety and let $q$ and $p$ be weights such that $q(z)=O(p(z))$ as $|z| \rightarrow \infty$. If the restriction map $R_{V}$ satisfies $A_{q}(V) \subset R_{V}\left(A_{p}(\mathbb{C})\right)$, then $R_{V}\left(A_{p}(\mathbb{C})\right)=A_{p}(V)$, i.e. $V$ is interpolating for $A_{p}(V)$.

Proof. The proof follows the steps of the proof of Theorem 1.2.7 in Section 1.2 of Ounaïes' thesis [19]. We first apply Lemma 2.1 to find $g_{0} \in A_{p}(\mathbb{C})$ such that $g_{0}^{(l)}\left(z_{1}\right)=0$ for $0 \leq l<m_{1}$, except $g_{0}^{\left(m_{1}-1\right)}\left(z_{1}\right)=1$ and $g_{0}^{(l)}\left(z_{j}\right)=0$ for all $j>1$ and $0 \leq l<m_{j}$. The function $f(z):=\left(z-z_{1}\right) g_{0}(z), z \in \mathbb{C}$, belongs to $A_{p}(\mathbb{C})$ by conditions (w3) and (w4), $f \neq 0$, and the multiplicity variety $V$ is contained in the set of zeros $Z(f)$ of $f$. We can now apply Jensen's formula, as in the proof of [19, Lemma 1.2.2], to conclude that $N_{V}(r, 0)=O(p(r))$ as $r \rightarrow \infty$; i.e. condition (a) (i) in Theorem 1.1 is satisfied.

Now we check that condition (a) (ii) in Theorem 1.1 also holds. To do that, we apply Lemma 2.1 to find the sequence $\left(f_{k}\right)_{k} \subset A_{p}(\mathbb{C})$ satisfying the conditions stated there. Set $g_{k}(z):=\left(z-z_{k}\right) f_{k}(z), z \in \mathbb{C}, k \in \mathbb{N}$. By conditions (w3) and (w4), there is a constant $B$, not depending on $k$, such that $\left|g_{k}(z)\right| \leq B \exp \left(B\left(p(z)+p\left(z_{k}\right)\right)\right)$ for each $z \in \mathbb{C}$ and each $k \in \mathbb{N}$. 
If $\left|z-z_{k}\right| \leq\left|z_{k}\right|$, we have $p(z) \leq p\left(2\left|z_{k}\right|\right) \leq C p\left(z_{k}\right)+C$ for some constant $C>0$ by condition (w4). Therefore we can apply Jensen's formula to $g_{k}(z) /\left(z-z_{k}\right)^{m_{k}}$ in the disc of center $z_{k}$ and radius $\left|z_{k}\right|$ as in the proof of [19, Theorem 1.2.7] to conclude that there is $D>0$ such that $N_{V}\left(z_{k},\left|z_{k}\right|\right) \leq D p\left(z_{k}\right)+D$ for all $k \in \mathbb{N}$, and $N_{V}\left(z_{k},\left|z_{k}\right|\right)=O\left(p\left(z_{k}\right)\right)$ as $k \rightarrow \infty$.

In the proof of Theorem 2.4 below we will apply [3, Corollary 4.11]: If a multiplicity variety $V=\left\{\left(z_{k}, m_{k}\right) \mid k \in \mathbb{N}\right\}$ satisfies $\left|z_{k+1}\right| \geq L\left|z_{k}\right|, k \in \mathbb{N}$, for some constant $L>1$, then $V$ is interpolating for $A_{p}(\mathbb{C})$ if and only if $N_{V}(r, 0)=O(p(r))$ as $r \rightarrow \infty$ and $m_{k} \log \left|z_{k}\right|=O\left(p\left(z_{k}\right)\right)$ as $k \rightarrow \infty$.

Lemma 2.3 If $p$ is a weight such that $p(t)=0$ for each $t \in[0,1]$, then the function $p(t) / \log t$ is non-decreasing and tends to $\infty$ as $t \rightarrow \infty$.

Proof. The convergence of $p(t) / \log t$ to $\infty$ as $t \rightarrow \infty$ follows from condition (w3) of the weight. On the other hand, since $p$ is radial, continuous and subharmonic by conditions (w1) and (w2), the function $\varphi(t):=p\left(e^{t}\right), t \in[0, \infty[$ is increasing, convex and satisfies $\varphi(0)=0$. We can apply a well-known result, see e.g. [20, Lemma 4.42], to conclude that $\varphi(t) / t, t \geq 0$, is non-decreasing. This implies the conclusion.

Theorem 2.4 If $q$ and $p$ are weights such that $q(z)=o(p(z))$ as $|z| \rightarrow \infty$, then there is a multiplicity variety $V=\left\{\left(z_{k}, m_{k}\right) \mid k \in \mathbb{N}\right\}$ such that $V$ is interpolating for $A_{p}(\mathbb{C})$, but not for $A_{q}(\mathbb{C})$.

Proof. Passing to an equivalent weight, we may assume without loss of generality that $p(t)=0$ for each $t \in[0,1]$. Therefore Lemma 2.3 implies that $p(t) / \log t$ is non-decreasing. We can also assume, passing again to an equivalent weight, that $q \leq p$. We start with the construction of the multiplicity variety by selecting $r_{1}>e$ such that $q\left(r_{1}\right)>1$, and we set $m_{1}:=\left[q\left(r_{1}\right)\right]$, where $[\alpha]$ denotes the integer part of the real number $\alpha \geq 0$. Now find $r_{2}>e r_{1}$ such that $\frac{q\left(r_{2}\right)}{\log r_{2}}>2 p\left(r_{1}\right)$. This is always possible by condition (w3) for the weight $q$. Now we select the multiplicity $m_{2}:=\left[\frac{p\left(r_{2}\right)}{\log r_{2}}\right]-m_{1}$. Since

$$
\frac{p\left(r_{2}\right)}{\log r_{2}} \geq \frac{q\left(r_{2}\right)}{\log r_{2}}>2 p\left(r_{1}\right)>m_{1}+1,
$$

we have $m_{2}>0$. Proceeding by induction, suppose that $r_{1}<\ldots<r_{k}$ and $m_{1}, \ldots, m_{k}$ are already selected. We first apply condition (w3) for the weight $q$ and the assumption $q=o(p)$ to select $r_{k+1}>e r_{k}$ such that

$$
\frac{q\left(r_{k+1}\right)}{\log r_{k+1}}>2 p\left(r_{k}\right) \text { and } p\left(r_{k+1}\right) \geq 2^{k+1} q\left(r_{k+1}\right) .
$$

We set

$$
m_{k+1}:=\left[\frac{p\left(r_{k+1}\right)}{\log r_{k+1}}\right]-\left[\frac{p\left(r_{k}\right)}{\log r_{k}}\right]
$$

As

$$
\frac{p\left(r_{k+1}\right)}{\log r_{k+1}} \geq \frac{q\left(r_{k+1}\right)}{\log r_{k+1}}>2 p\left(r_{k}\right)>2 \frac{p\left(r_{k}\right)}{\log r_{k}}
$$


we conclude $m_{k+1}>0$. We now show that the multiplicity variety $V=\left\{\left(r_{k}, m_{k}\right) \mid k \in \mathbb{N}\right\}$ satisfies the desired properties. We first prove that

$$
\left(m_{1}+\ldots+m_{k}\right) \leq p(r), r_{k} \leq r<r_{k+1}, k \in \mathbb{N} .
$$

Indeed, for $r_{k} \leq r<r_{k+1}$, we have

$$
\left(m_{1}+\ldots+m_{k}\right) \leq\left[\frac{p\left(r_{k}\right)}{\log r_{k}}\right] \leq \frac{p(r)}{\log r} .
$$

Here the last inequality follows because $p(t) / \log t$ is non-decreasing. In particular we have $m_{k} \log r_{k} \leq p\left(r_{k}\right)$ for each $j \in \mathbb{N}$. On the other hand, it is easy to see from the definition of the integrated counting function that, for $r_{k} \leq r<r_{k+1}, N_{V}(r, 0) \leq\left(m_{1}+\ldots+m_{k}\right) \log r$. Therefore $N_{V}(r, 0) \leq p(r)$ for each $r \geq r_{1}$. By construction $r_{k+1}>e r_{k}$ for each $k \in \mathbb{N}$, hence we can apply [3, Corollary 4.11] to conclude that $V$ is interpolating for $A_{p}(\mathbb{C})$. It remains to prove that $V$ is not interpolating for $A_{q}(\mathbb{C})$. To see this, observe that for each $k \in \mathbb{N}$ we have

$$
\frac{m_{k} \log r_{k}}{q\left(r_{k}\right)} \geq \frac{p\left(r_{k}\right)}{q\left(r_{k}\right)}-\frac{\log r_{k}}{q\left(r_{k}\right)}-\frac{p\left(r_{k-1}\right)}{\log r_{k-1}} \frac{\log r_{k}}{q\left(r_{k}\right)} .
$$

By our inductive construction, this implies

$$
\frac{m_{k} \log r_{k}}{q\left(r_{k}\right)} \geq 2^{k}-\frac{\log r_{k}}{q\left(r_{k}\right)}-\frac{1}{2 \log r_{k-1}},
$$

that tends to infinity as $k \rightarrow \infty$ by condition (w3) of the weight $q$. We can apply again [3, Corollary 4.11] to conclude that $V$ is not interpolating for $A_{q}(\mathbb{C})$.

\section{Subspaces of the restriction map on $A_{p}^{0}(\mathbb{C})$}

Proposition 3.1 Let $V=\left\{\left(z_{k}, m_{k}\right) \mid k \in \mathbb{N}\right\}$ a multiplicity variety and let $q$ and $p$ be weights such that $q(z)=O(p(z))$ as $|z| \rightarrow \infty$. If the restriction map $R_{V}$ satisfies $A_{q}^{0}(V) \subset R_{V}\left(A_{p}^{0}(\mathbb{C})\right)$, then $R_{V}\left(A_{p}^{0}(\mathbb{C})\right)=A_{p}^{0}(V)$, i.e. $V$ is interpolating for $A_{p}^{0}(V)$.

Proof. We consider on $R_{V}\left(A_{p}^{0}(\mathbb{C})\right)$ the quotient topology $\tau$ with respect to the surjective linear map $R_{V}: A_{p}^{0}(\mathbb{C}) \rightarrow R_{V}\left(A_{p}^{0}(\mathbb{C})\right)$. The space $\left(R_{V}\left(A_{p}^{0}(\mathbb{C})\right), \tau\right)$ is a Fréchet space by [16, Proposition 25.3] with a topology finer than the one induced by $A_{p}^{0}(V)$. By assumption $A_{q}^{0}(V) \subset R_{V}\left(A_{p}^{0}(\mathbb{C})\right)$. The inclusion $i: A_{q}^{0}(V) \rightarrow\left(R_{V}\left(A_{p}^{0}(\mathbb{C})\right), \tau\right)$ has closed graph, since the inclusion $i: A_{q}^{0}(V) \rightarrow A_{p}^{0}(V)$ is continuous. Hence, by the closed graph theorem [16, Theorem 8.8], $i: A_{q}^{0}(V) \rightarrow\left(R_{V}\left(A_{p}^{0}(\mathbb{C})\right), \tau\right)$ is continuous. Let $B$ be a bounded subset of $A_{q}^{0}(V)$. Since $A_{q}^{0}(V)$ is nuclear, in particular Montel, $B$ is relatively compact in $\left(R_{V}\left(A_{p}^{0}(\mathbb{C})\right), \tau\right)$. By [16, Corollary 26.22], there is a compact, hence bounded, subset $C$ of $A_{p}^{0}(\mathbb{C})$ such that $B \subset R_{V}(C)$. Accordingly, selecting an appropriate bounded sequence in $A_{q}^{0}(V)$ of elements with coordinates $0^{\prime} s$ and $1^{\prime} s$, we find a bounded sequence $\left(f_{k}\right)_{k} \subset A_{p}^{0}(\mathbb{C})$ such that $f_{k}^{(l)}\left(z_{j}\right)=0,0 \leq l<m_{j}$, for each $k$ and $j$, except $f^{\left(m_{k}-1\right)}\left(z_{k}\right) /\left(m_{k}-1\right) !=1$. Since the sequence is bounded in $A_{p}^{0}(\mathbb{C})$, for each $n \in \mathbb{N}$ there is $A_{n}>0$ such that

$$
\left|f_{k}(z)\right| \leq A_{n} \exp \left(\frac{1}{n} p(z)\right), \quad z \in \mathbb{C}, k \in \mathbb{N} .
$$


Setting $g_{k}(z):=\left(z-z_{k}\right) f_{k}(z), k \in \mathbb{N}$, we can apply condition (w3) of the weight to conclude that for each $n \in \mathbb{N}$ there is $B_{n}>0$ such that

$$
\left|f_{k}(z)\right| \leq B_{n} \exp \left(\frac{1}{n} p(z)+\frac{1}{n} p\left(z_{k}\right)\right), \quad z \in \mathbb{C}, k \in \mathbb{N} .
$$

After these preparations, it is possible to proceed similarly as we did in the proof of Proposition 2.2 to conclude that $V$ satisfies conditions (b) (i) and (ii) of Theorem 1.1, thus $V$ is interpolating for $A_{p}^{0}(V)$.

Theorem 3.2 If $q$ and $p$ are weights such that $q(z)=o(p(z))$ as $|z| \rightarrow \infty$, then there is a multiplicity variety $V=\left\{\left(z_{k}, m_{k}\right) \mid k \in \mathbb{N}\right\}$ such that $V$ is interpolating for $A_{p}^{0}(\mathbb{C})$, but not for $A_{q}^{0}(\mathbb{C})$.

Proof. Proceeding as in the proof of [15, Lemma 2.3] (see also [9, Lemmas 1.6 and 1.7]) we can find another weight $m$ such that $q(z)=o(m(z))$ and $m(z)=o(p(z))$ as $|z| \rightarrow \infty$. By Theorem 2.4 there is a multiplicity variety $V$ which is interpolating for $A_{m}(\mathbb{C})$ but not for $A_{q}(\mathbb{C})$. Since $m(z)=o(p(z))$ as $|z| \rightarrow \infty$, we can apply Theorem 1.1 (a) and (b) to conclude that $V$ is interpolating for $A_{p}^{0}(\mathbb{C})$. On the other hand, as $V$ is not interpolating for $A_{q}(\mathbb{C})$, it follows again from Theorem 1.1 that it is not interpolating for $A_{q}^{0}(\mathbb{C})$.

We conclude this paper utilizing a reduction argument of Meise and Taylor [15] to obtain a consequence of the main result of [18] on the description of the range $R_{V}\left(A_{p}^{0}(\mathbb{C})\right)$ of the restriction operator on $A_{p}^{0}(\mathbb{C})$ in terms of divided differences. To do this, let $V=\left\{\left(z_{k}, m_{k}\right) \mid k \in\right.$ $\mathbb{N}\}$ be a multiplicity variety and let $p$ be a weight.

Given a sequence $W:=\left(w_{k, l}\right)_{k \in \mathbb{N}, 0 \leq l<m_{k}}$ of complex numbers, we denote by $\Phi(W):=$ $\left(\phi_{k, l}\right)_{k \in \mathbb{N}, 0 \leq l<m_{k}}$ the divided differences of $W$ defined by induction as in [18]. We denote by $\tilde{A}_{p}^{0}(V)$ the set of all the sequences $W:=\left(w_{k, l}\right)_{k \in \mathbb{N}, 0 \leq l<m_{k}}$ such that their divided differences $\Phi(W):=\left(\phi_{k, l}\right)_{k \in \mathbb{N}, 0 \leq l<m_{k}}$ satisfy that for all $\varepsilon>0$ there is $A_{\varepsilon}>0$ such that for all $n \in \mathbb{N}$ and all $\left|z_{k}\right| \leq 2^{n}$ and $0 \leq l<m_{k}$ we have

$$
\delta_{k, l}:=\left|\phi_{k, l}\right| 2^{n\left(l+m_{1}+\ldots+m_{k-1}\right)} \leq A_{\varepsilon} \exp \left(\varepsilon p\left(2^{n}\right)\right) .
$$

Proposition 3.3 Let $p$ be a weight. Let $V=\left\{\left(z_{k}, m_{k}\right) \mid k \in \mathbb{N}\right\}$ be a multiplicity variety satisfying condition (b) (i) in Theorem 1.1, i.e. $N_{V}(r, 0)=o(p(r))$ as $r \rightarrow \infty$. Then the range $R_{V}\left(A_{p}^{0}(\mathbb{C})\right)$ of the restriction map on $A_{p}^{0}(\mathbb{C})$ coincides with $\tilde{A}_{p}^{0}(V)$.

Proof. Given $W \in \tilde{A}_{p}^{0}(V)$, for $2^{n} \leq R<2^{n+1}$, define

$$
g(R):=N_{V}(R, 0)+\log \left(\sup _{0 \leq l<m_{k},\left|z_{k}\right| \leq 2^{n}} \delta_{k, l}\right) .
$$

Then $g(R)=o(p(R))$ as $R \rightarrow \infty$, and we argue as in the proof of [15, Lemma 2.3] (see also $[9$, Lemmas 1.6 and 1.7]) to find a weight $q$ such that $g(R)=o(q(R))$ and $q(R)=o(p(R))$ as $R \rightarrow \infty$. By [18, Theorem 1.11] we have $W \in R_{V}\left(A_{q}(\mathbb{C})\right)$. Since $q(R)=o(p(R))$ as $R \rightarrow \infty$, $W \in R_{V}\left(A_{p}^{0}(\mathbb{C})\right)$. This yields $\tilde{A}_{p}^{0}(V) \subset R_{V}\left(A_{p}^{0}(\mathbb{C})\right)$. To prove the other inclusion, for each $f \in A_{p}^{0}(\mathbb{C})$ there is a weight $q$ such that $f \in A_{q}(\mathbb{C}), N_{V}(R, 0)=o(q(R))$ and $q(R)=o(p(R))$ as $R \rightarrow \infty$ (cf. [15, Lemma 2.3] and [9, Lemma 1.7]). We apply [18, Theorem 1.11] again to 
conclude $R_{V}(f) \in \tilde{A}_{q}(V) \subset \tilde{A}_{p}^{0}(V)$. This completes the description of the range in this case.

\section{References}

[1] C. A. Berenstein and R. Gay. Complex variables, volume 125 of Graduate Texts in Mathematics. Springer-Verlag, New York, 1991. An introduction.

[2] C. A. Berenstein and R. Gay. Complex analysis and special topics in harmonic analysis. Springer-Verlag, New York, 1995.

[3] C. A. Berenstein and Bao Qin Li. Interpolating varieties for spaces of meromorphic functions. J. Geom. Anal., 5(1):1-48, 1995.

[4] C. A. Berenstein, Bao Qin Li, and A. Vidras. Geometric characterization of interpolating varieties for the (FN)-space $A_{p}^{0}$ of entire functions. Canad. J. Math., 47(1):28-43, 1995.

[5] C. A. Berenstein and B. A. Taylor. A new look at interpolation theory for entire functions of one variable. Adv. in Math., 33(2):109-143, 1979.

[6] J. Bonet and A. Galbis. The range of non-surjective convolution operators on Beurling spaces. Glasgow Math. J., 38(1):125-135, 1996.

[7] J. Bonet, A. Galbis and S. Momm. Nonradial Hörmander algebras of several variables and convolution operators. Trans. Amer. Math. Soc., 353(6): 2275-2291, 2001.

[8] R. W. Braun. Weighted algebras of entire functions in which each closed ideal admits two algebraic generators. Michigan Math. J., 34(3):441-450, 1987.

[9] R. W. Braun, R. Meise, and B. A. Taylor. Ultradifferentiable functions and Fourier analysis. Results Math., 17(3-4):206-237, 1990.

[10] J. Buckley, X. Massaneda, and J. Ortega-Cerdà. Traces of functions in Fock spaces on lattices of critical density. Bull. Lond. Math. Soc., 44(2):222-240, 2012.

[11] A. Hartmann and X. Massaneda. On interpolating varieties for weighted spaces of entire functions. J. Geom. Anal., 10(4):683-696, 2000.

[12] X. Massaneda, J. Ortega-Cerdà, and M. Ounaïes. A geometric characterization of interpolation in $\hat{\mathcal{E}}^{\prime}(\mathbb{R})$, Trans. Amer. Math. Soc., 358: 3459-3472, 2006

[13] X. Massaneda, J. Ortega-Cerdà, and M. Ounaïes. Traces of Hörmander algebras on discrete sequences. In Analysis and mathematical physics, Trends Math., pages 397-408. Birkhäuser, Basel, 2009.

[14] R. Meise. Sequence space representations for (DFN)-algebras of entire functions modulo closed ideals. J. Reine Angew. Math., 363:59-95, 1985.

[15] R. Meise and B. A. Taylor. Sequence space representations for (FN)-algebras of entire functions modulo closed ideals. Studia Math., 85(3):203-227, 1987. 
[16] R. Meise and D. Vogt. Introduction to functional analysis, volume 2 of Oxford Graduate Texts in Mathematics. The Clarendon Press Oxford University Press, New York, 1997. Translated from the German by M. S. Ramanujan and revised by the authors.

[17] M. Ounaïes. Geometric conditions for interpolation in weighted spaces of entire functions. J. Geom. Anal., 17(4):701-715, 2007.

[18] M. Ounaïes. Interpolation by entire functions with growth conditions. Michigan Math. J., 56(1):155-171, 2008.

[19] M. Ounaïes. Interpolation dans les algèbres de Hörmander. Université Louis Pasteur. Institut de Recherche Mathématique Avancée (IRMA), Strasbourg, 2008.

[20] K. R. Stromberg. Introduction to classical real analysis. Wadsworth International, Belmont, Calif., 1981. Wadsworth International Mathematics Series.

[21] P. Zioło. Geometric characterization of interpolation in the space of Fourier-Laplace transforms of ultradistributions of Roumieu type. Collect. Math., 62(2):161-172, 2011.

\section{Authors' addresses:}

José Bonet: Instituto Universitario de Matemática Pura y Aplicada IUMPA, Universitat Politècnica de València, E-46071 Valencia, Spain

email:jbonet@mat.upv.es

Carmen Fernández: Departamento de Análisis Matemático, Universidad de Valencia, E46100 Burjasot

email:fernand@uv.es 